\title{
FACTOR ANALYSIS OF UPPER LIMB PROSTHETIC ACCEPTANCE FROM RETROSPECTIVE PROSTHETIC CLINICIAN SURVEY
}

\author{
Gerald E. Stark \\ School of Biological Sciences, Georgia Institute of Technology, Georgia, USA. \\ Email: gerald.e.stark@gmail.com
}

DOI: https://doi.org/10.33137/cpoj.v1i2.32045

\section{INTRODUCTION}

Upper limb prosthetic acceptance seems to be relatively unchanged from 1958 where it was measured to be $75 \%$ for transradial, $61 \%$ for transhumeral, and $35 \%$ for shoulder disarticulation levels. A practitioner survey from 2013 by the author found this to be largely unchanged at $79.6 \%, 57.8 \%$, and $32.8 \%$ respectively. An upper limb meta-analysis showed that the most significant factors affecting prosthetic rejection using a median rating were function, comfort, ease of use, weight, heat, lack of sensory feedback, inconvenience, lifestyle, dissatisfaction with technology, irritation, and availability of services. An earlier survey by the author condensed these factors of rejection to amputation level, functional advantage, and comfort, and included confidence of the prosthetist, availability of therapy, and support of the patient context. Also it was speculated that the value of factors influencing rejection of prostheses may not be simply the converse of those accepting the prosthesis but different scales.

\section{METHODS}

A retrospective case survey was created using a thirdparty web-based survey provider that was posted from 3/15/17 to 4/1/17 using postings on the OANDP ListServ to recruit participants. Prosthetists were asked to recall up to their last three upper limb fittings. There were 75 respondents with 209 retrospective cases. Based on the number of UL fitting, participants were participants classified: 12 specialists, 20 experts, 27 intermediates, and 16 novices. The participants were asked to assess various retrospective factors including prosthetic level, type of control, patient gadget tolerance, patient functional expectation, comfort tolerance, patient value of cosmesis, confidence of prosthetist, availability of therapy, experience level of therapist, daily wear time, patient description of prosthesis, and patient assessment of cosmetic quality. By level the distribution was 24 finger/transcarpal, 17 wrist disarticulation, 110 transradial, 4 elbow disarticulation, 47 transhumeral, 6 shoulder disarticulation, and 1 interscapular thoracic reflecting a common distribution. Body power control predominated with 84 and external power as 72 with passive at 27 , and hybrid control at 26 .

\section{RESULTS}

The clinician self-assessments predominantly showed highly favorable, and perhaps skewed, outcomes with respect to functional expectation, gadget tolerance, prosthetic confidence, contextual support, patient wear time, patient proficiency, cosmetic acceptance, and description of the prosthesis. Areas of more normalized distribution appeared to be upper limb experience for therapists, patient experience, and prosthetic description. Using Pearson's coefficient, there were statistically significant relationships at $\mathrm{p}$ (one-tailed)<.01 level between the patient acceptance level and patient experience, gadget tolerance, functional expectation, comfort tolerance, prosthetist confidence, and patient cosmetic description and a negative $\mathrm{p}<.05$ relationship with experience of the therapist. The relationships were true of the number of hours worn. A very strongly predictive relationship of $\mathrm{R} 2$ Linear $=.564 \mathrm{~F}(10,197)=$ 25.513 with all of the factors listed above. However a systematic multi-variable reduction found a strong significance, $\mathrm{R} 2$ Linear $=.556, \mathrm{~F}(3,204)=85.302$, with the patient acceptance level, gadget tolerance, patient experience, which may not have practical clinical relevance.

\section{DISCUSSION}

The skewed distribution of prosthetist self-assessment for functional expectation, gadget tolerance, comfort, prosthetic confidence, contextual support, and patient proficiency, wear time, and prosthetic description of this retrospective may be indicative of a group halo or optimism bias. Also this calls into question the validity and reliability of the sample and relevance to the application. However, the negative relationship between the expertise of therapist and proficiency of the patient may indicate that the therapist may mitigate this bias and 
influence a clearer understanding of functional outcome. The strong statistically predictive relationship between the 10 identified factors suggests these may provide an indicator of acceptance. However additional direct patient understanding seems to be needed to draw a narrower focus as to the most significant factors for clinical use.

\section{CONCLUSION}

More research needs to be done directly done with upper limb prosthetic users with respect to acceptance rather than the convenience sample of prosthetists. As a group, clinicians seem to present a higher assessment of their abilities due to an optimism bias. External evaluation by a therapist seems crucial to understanding true patient functional performance However, this current work may help to establish which factors seem to have the greatest relationship for acceptance for subsequent research.

\section{REFERENCES}

Berger, N. Studies of the upper extremity amputee II. The population (1953-55) Artificial Limbs. 1958; 5 (1), p. 57-72.

Biddess, E., Chau, T. (2007). Upper limb prosthetics: Critical factors in device abandonment. Am J Phys Med Rehabil. 2007; 86, p. 977-987. DOI:10.1097/PHM.0b013e3181587f6c

Stark, G. Factor analysis of upper extremity prosthetic patient acceptance. Paper presented at the American Orthotic and Prosthetic Association National Meeting, Las Vegas, Nevada, 2014.

Burrough, S., Brook, J. Patterns of acceptance and rejection of upper limb prostheses. Orthotics and Prosthetics, 1985, 39(2), p. 40-47. 